OPEN ACCESS

Edited by:

Jianying Zhang,

The University of Texas at El Paso,

United States

Reviewed by:

Yi Zhang,

First Affiliated Hospital of Zhengzhou

University, China

Jessica Fioravanti,

Regensburg Center for Interventional

Immunology (RCI), Germany

*Correspondence:

Jie Shen

shenjie2008nju@163.com

Baorui Liu

baoruiliu@nju.edu.cn

${ }^{\dagger}$ These authors have contributed equally to this work

Specialty section:

This article was submitted to Cancer Immunity and Immunotherapy,

a section of the journal

Frontiers in Immunology

Received: 24 March 2021 Accepted: 27 May 2021

Published: 13 July 2021

Citation

Liu C, Shao J, Dong Y, Xu Q

Zou Z, Chen F, Yan J, Liu J,

Li S, Liu B and Shen J (2021)

Advanced HCC Patient Benefit From

Neoantigen Reactive T Cells Based

Immunotherapy: A Case Report.

Front. Immunol. 12:685126.

doi: 10.3389/fimmu.2021.685126

\section{Advanced HCC Patient Benefit From Neoantigen Reactive T Cells Based Immunotherapy: A Case Report}

\author{
Chenxi Liu ${ }^{\dagger}$, Jie Shao ${ }^{\dagger}$, Yanbing Dong, Qiuping Xu, Zhengyun Zou, Fangjun Chen, \\ Jing Yan, Juan Liu, Shuangshuang Li, Baorui Liu* and Jie Shen* \\ Comprehensive Cancer Centre of Drum Tower Hospital, Medical School of Nanjing University, Clinical Cancer Institute of \\ Nanjing University, Nanjing, China
}

Advanced hepatocellular carcinoma $(\mathrm{HCC})$ is a highly lethal disease, mainly due to the late stage at diagnosis and its rapid progression. Although patients with advanced HCC can choose targeted therapy or chemotherapy, overall, the treatment response rate is extremely low and the average survival time is one year more or less. But the application of immunotherapy have led to a paradigm shift in the treatment of HCC, such as TILs (tumor infiltrating lymphocytes), Checkpoint blockade (immune Checkpoint blockade), CAR-T(chimeric antigen receptor T cells) and TCR-T (engineered t-cell receptor T cells). And recent data indicate neoantigens generated when tumors mutate are the main target of tumor-specific TILs, and they are also the main antigens mediating tumor regression in TILs treatment. Moreover, numerous evidences have revealed that radiotherapy lead to massive release of tumor antigens, which may increase the effectiveness of immunotherapy. Based on the above theory, we used neoantigen reactive $T$ cells combined with tomotherapy to treat a patient with advanced $\mathrm{HCC}$ (Clinical Trial Study Registration Number: NCT03199807), who reached a long time progress free survival.

Keywords: hepatocellular carcinoma, neoantigen reactive $\mathrm{T}$ cells, immunotherapy, tomotherapy, benefit

\section{INTRODUCTION}

In China, hepatocellular carcinoma is among the top five cancer types that have high morbidity and mortality (1). Approximately $80 \%$ of the HCC patients were initially diagnosed in an advanced or metastatic stage. Surgery is the first treatment for HCC patients, but not many patients are eligible for surgery, and the 5 -year risk of recurrence after hepatectomy is as high as $50 \%-70 \%(2,3)$. Interventional 
therapy and radiotherapy are mostly used as auxiliary treatment methods. In terms of targeted therapy or chemotherapy, the average survival time of patients receiving this treatment is one year more or less, and the remission rate is $2 \%-18.8 \%(4-7)$. Although the use of PD-1 can effectively prolong the survival of patients, the response rate of single-drug use is no more than 16$20 \%$, this suggests that the use of single drugs needs to screen effective populations. In summary, looking for more effective immunotherapy may become a new breakthrough in the field of current HCC treatment.

In a clinical trial of metastatic melanoma, Dr. Rosenberg and his colleagues, who worked at NCI in the United States, confirmed that TILs back-transfusion combined with nonmyeloablative chemotherapy or radiotherapy could achieve a clinical remission rate of $40 \%-72 \%$, and nearly $40 \%$ of patients with complete remission (CR) showed no recurrence for more than 7 years (8). The above data indicate that TILs may bring new hope for the treatment of HCC, and the neoantigens that are the main targets of TILs, with its unique advantages, it has become a hot field in today's anti-tumor research.

Neoantigens are a class of antigens derived from normal human genomes. Antigens produced by mutant proteins and oncoviruses are integrated into human genome. They have high affinity with TCR and strong immunogenicity. Compared with the traditional tumor-associated antigen (TAA), neoantigen is not expressed by normal tissues; and thereby, it does not cause central immune tolerance or autoimmune diseases (9). In addition, in 2015, Schumacher TN et al. conducted a tumor mutation spectrum analysis on several common solid tumor types, and showed that the mutation frequency of liver cancer was among the highest (10), and radiotherapy lead to massive release of tumor antigens (11). Based on the above principles, neoantigen reactive $\mathrm{T}$ cells combined with tomotherapy have been considered as a promising therapy for advanced cancer. In our previous study, we have combined TCGA data and the NGS sequencing results of the liver cancer samples from our center to screen out the genes with high-frequency mutations (12). Based on the common HLA genotyping (HLA- $\mathrm{a}^{\star} 02: 01$, HLA- $\mathrm{a}^{\star} 02: 03$, HLA-a $\left.{ }^{\star} 02: 06, H L A-a^{\star} 11: 01, H L A-a^{\star} 24: 02\right)$ in Chinese population, 29 epigenetic peptides were selected and validated the antigenicity and other related parameters of the library (12), and finally we further conducted the clinical study that combined neoantigen reactive $\mathrm{T}$ cells with tomotherapy (Clinical Trial Study Registration Number: NCT03199807). Here, we reported a case that had significantly beneficial results from this therapy. Written informed consent was obtained from the individual for the publication of any potentially identifiable images or data included in this article.

\section{CASE PRESENTATION}

The patient was male, 75-year-old, with a history of chronic hepatitis B. Partial hepatectomy was performed after the diagnosis of primary liver cancer in Apr-2016. In Oct-2016,abdominal Computed Tomography (CT) re-examination revealed multiple enhanced nodules in the liver, and the possibility of metastasis was considered. Therefore, this patient underwent 3 Transarterial Chemoembolization (TACE) operations.

Unfortunately, the abdominal Magnetic Resonance Imaging (MRI) re-examination in Feb-2017 revealed multiple metastatic liver lesions with enlarged hilar lymph nodes.

The patient strongly requested to participate in our clinical trial of tomotherapy combined with Neoantigen Reactive T (NRT) Cells based immunotherapy. In March 2017, this patient received tomotherapy for local lesions in the right anterior lobe of the liver; the cumulative dose was: Planning Target Volume (PTV): 50Gy/ 10f. After tomotherapy, the patient received personalized NRT immune cells reinfusion combined with IL-2(400WU/d, civ, 5 days in total) and GM-CSF cytokine (150ug/d, ih, 5 days in total) therapy in March 2017, with one month per cycle and a total of four cycles. According to the patient gene expression profile, the neo-antigen was chosen as: (HLA-a $\left.{ }^{\star} 11: 01\right)$ : KRAS-(G12A) p0716: VVVGAAGVGK; KRAS-(G13D) p07-16: VVVGAGDVGK; PIK3CA-(H1047L) p1046-1054: ALHGGWTTK; IDH1(R132H) p123-142: GWVKPIIIGHHAYGDQYRAT.

This patient was PD-L1(+), and TMB-H. The re-examination after 2 cycles of treatment showed that the lesion on the right anterior lobe of the liver became smaller, and the number of nodules in the more enhanced focus was reduced. The tumor markers were significantly down-regulated, and the changes in HCC specific tumor marker AFP were listed in Figure 1A. At the same time, the lymph number gradually increased, and the changes in lymphocytes after per cycle of NRT treatment were shown in Figure 1B. After four cycle of NRT, the overall efficacy evaluation was $\mathrm{SD}$, and only regular examination was continued. The amount of $\mathrm{T}$ cells reinfusion per cycle and the amount of NRT immune cells detected in-vivo were listed in Table 1. Except for the fever during the treatment (the highest temperature was $38.3^{\circ} \mathrm{C}$, and it was relieved after Paracetamol), there was no other discomfort or obvious liver toxicity.

10 months after the radiotherapy combined with personalized cellular immunotherapy, a review of the abdominal MRI on May 2018 showed that the left outer lobe of the liver appeared, which was considered as disease progression. After full informed consent, the patient was enrolled in the RESCUE project (13) with combined PD-1 antibody and apatinib. From 2018-6, the patient received intravenous injection of PD-1 antibody(SHR$1210,200 \mathrm{mg} / \mathrm{q} 2 \mathrm{w}$,iv, two times as a cycle) and apatinib $(250 \mathrm{mg} / \mathrm{d}$, po). After 2 cycles of PD-1 antibody treatment, the reexamination showed that the intrahepatic mass of porta hepatis and left outer lobe were significantly smaller than before, with the overall efficacy evaluation as partial response (PR). However, due to the hand-foot syndrome side-effect of apatinib, which seriously affected the daily activities of patient, the patient stopped taking apatinib from 2018-7. Therefore, since then, PD-1 antibody single-drug treatment was performed till 2020-6, lasting for a total of 2 years. The patient has been in a fairly good condition. The regular reviews of the tumor showed gradually shrinking of lesion in porta hepatis, and complete response (CR) of intrahepatic lesions in left outer lobe. The patient's imaging changes were shown in Figure 2. The target 


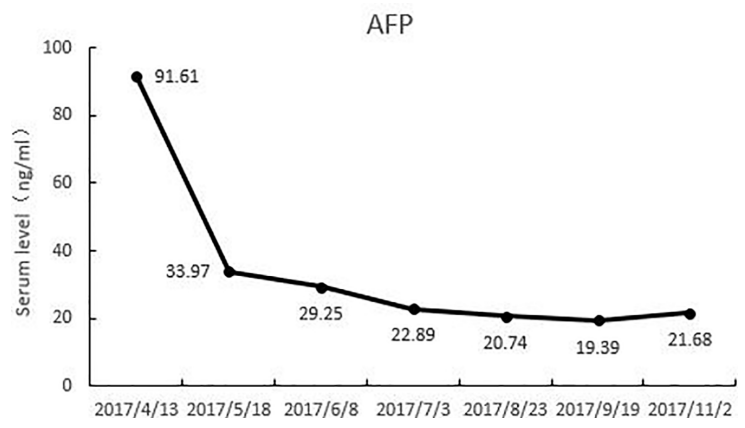

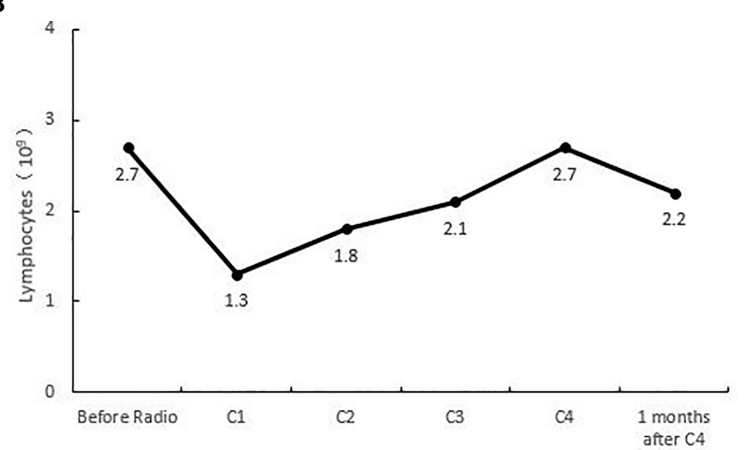

FIGURE 1 | (A) alpha fetoprotein (AFP) levels of this patient during the treatment of tomotherapy combined with Neoantigen Reactive T Cells. (B) The lymphocyte levels of this patient during the treatment of tomotherapy combined with Neoantigen Reactive T Cells.

TABLE 1 | The amount of T cells reinfusion per cycle and the amount of NRT immune cells detected in-vivo.

\begin{tabular}{|c|c|c|c|}
\hline & Number of NRT $\left(10^{10}\right)$ & $\mathrm{CD}^{+}{ }^{\mathrm{CD}} 4^{+} \mathrm{CD} 137^{+}\left(10^{8}\right)$ & $\mathrm{CD}^{+} \mathrm{CD}^{+} \mathrm{CD} 137^{+}\left(10^{8}\right)$ \\
\hline Second cycle & 2.04 & $1.02(0.5 \%)$ & 3.47 (1.7\%) \\
\hline Fourth cycle & 1.12 & $0.78(0.7 \%)$ & $2.13(1.9 \%)$ \\
\hline
\end{tabular}

lesion changes at different time points during and after the treatment were listed in Table 2.In order to intuitively reflect the patient's prognosis and clinical curative effect, we listed the entire treatment process in Figure 3.

\section{DISCUSSION}

This is a case of comprehensive treatment with obvious benefits. In the first stage, the patient benefited from tomotherapy combined with Neoantigen Reactive T Cells. The lesion with radiotherapy was significantly reduced, and the remaining lesions were stable. In the second stage, new lesion appeared on the left lobe, and the patient benefited from the treatment of apatinib combined with PD-1 antibody. At the final stage, PD-1 antibody maintenance treatment was going on after the abandonment of apatinib because of the serious side effects, this patient continued to get benefit from treatment. From the genetic test, this patient is positive for TMB-H and PD-L1, which may be a potential predictor of his benefit from immunotherapy. At the same time, this patient has mutations in KRAS and IDH1, which are frequent mutation sites in HCC, providing a genetic basis for neoantigen immunotherapy. The patient's early cell therapy provides a material basis for the subsequent PD-1 antibody to a certain extent.

In this comprehensive treatment, it reflects the synergy of radiotherapy and immunotherapy. In recent years, the synergistic effect of radiation and immunotherapy has been reported widely. Radiation can cause tumor to release tumor antigens, transforming cold tumors into hot tumors. And these changes in immune microenvironment can attract $\mathrm{T}$ cells to attack the tumor site, thus synergizing the effects of radiation and immune response (14-16). In addition, radiotherapy can lead to a significant decrease in lymphocyte count, which possibly eliminates the inhibitory or dysfunctional $\mathrm{T}$ cells. We also observed this change in our study. The number of lymphocytes in patients declined significantly after radiotherapy. But after we transfused the $\mathrm{T}$ cells back into patients, the number of lymphocytes gradually increased and played a synergistic antitumor role.

In this comprehensive treatment, it also reflects the synergy of anti-angiogenesis and immunotherapy. Studies have shown that the basic principle of anti-angiogenic drugs combined with immunotherapy is that anti-angiogenic drugs can inhibit the occurrence of angiogenesis and immune escape in the tumor microenvironment, at the same time, this effect can enhance the body's immune response to achieve anti-tumor effects $(17,18)$. The Keynote524 study also shows that when the two drugs are used in combination, the objective response rate(ORR) of patients with advanced HCC can reach $36 \%$, progression-free survival (PFS) reaches 8.6 months, and overall survival (OS) reaches 22 months (19). We also observed a significant reduction of the lesion in the study. After the patient's left outer lobe of the liver was treated with anti-angiogenesis combined immunotherapy, the lesion was significantly reduced, and the efficacy was evaluated as PR.

Overall, this comprehensive treatment model is safe and well tolerated. Except for hand-foot syndrome caused by apatinib, 
right anterior lobe of the liver:

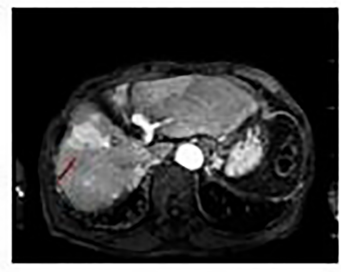

(a)17-02-27

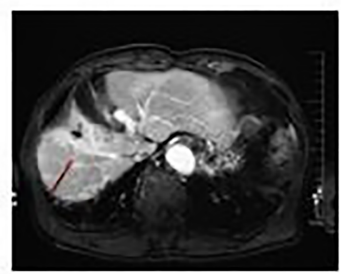

(d) $18-05-21 \mathrm{SD}$

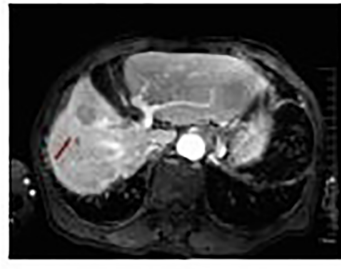

(b)17-5-20 PR

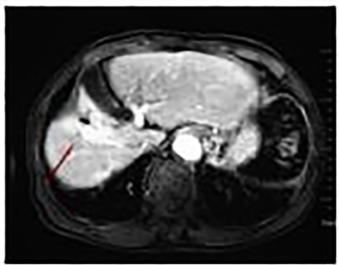

(e)18-08-06 SD

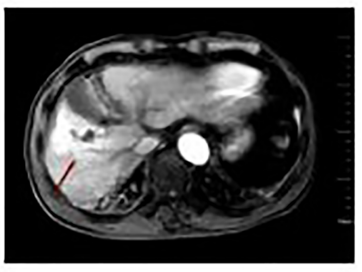

(c) $17-08-23 \mathrm{PR}$

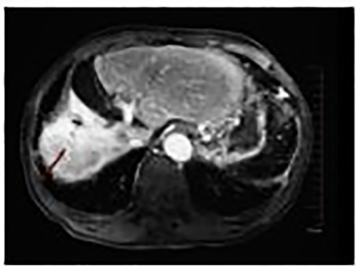

(f) $18-11-26 \mathrm{SD}$

porta hepatis:

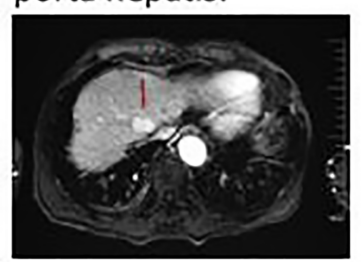

(g)17-02-27

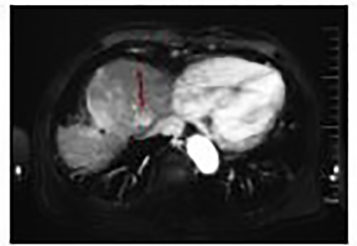

(j)18-05-21 SD

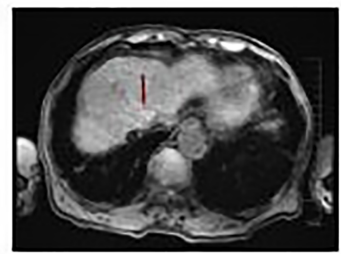

(h) 17-5-20 SD

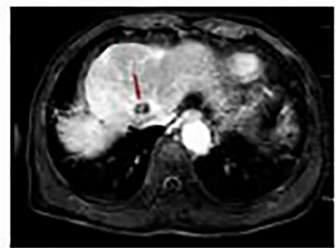

(k)18-08-06 PR

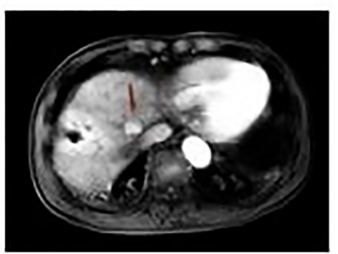

(i) 17-08-23 SD

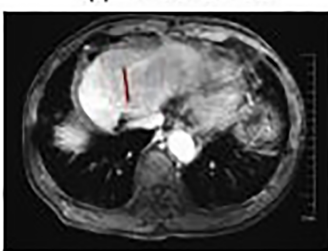

(I)18-11-26 PR

left outer lobe of the liver:

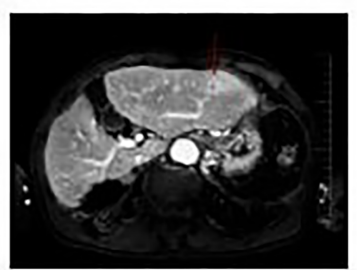

(m)18-05-21 PD

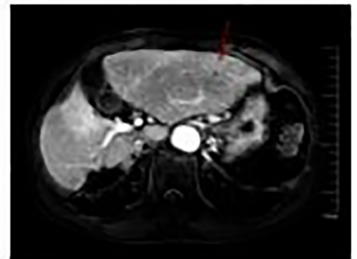

(n)18-08-06 PR

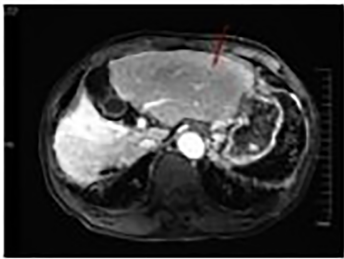

(o)18-11-26 CR

FIGURE 2 | Plain and enhanced MRI of lesions in different parts of patient in different periods. a-f: right anterior lobe of the liver; g-l: porta hepatis; m-o: left outer lobe of the liver. Efficacy evaluation: PD, Disease progression; SD, Stable disease; PR, Partial response; CR, Complete response. 
TABLE 2 | The target lesion changes at different time points during and after the treatment.

\begin{tabular}{|c|c|c|c|c|c|c|c|}
\hline & $2017-2(\mathrm{~mm})$ & 2017-8 (mm) & 2018-5 (mm) & 2018-8 (mm) & $2018-11(\mathrm{~mm})$ & 2020-07 (mm) & $2021-3(\mathrm{~mm})$ \\
\hline right anterior lobe & 33.60 & 22.93 & 18.45 & 18.65 & 18.60 & 18.62 & 18.69 \\
\hline porta hepatis & 21.48 & 18.35 & 19.08 & 7.71 & 9.25 & 6.21 & 6.21 \\
\hline left outer lobe & none & none & 18.00 & 7.11 & 0 & 0 & 0 \\
\hline
\end{tabular}

7.71

0

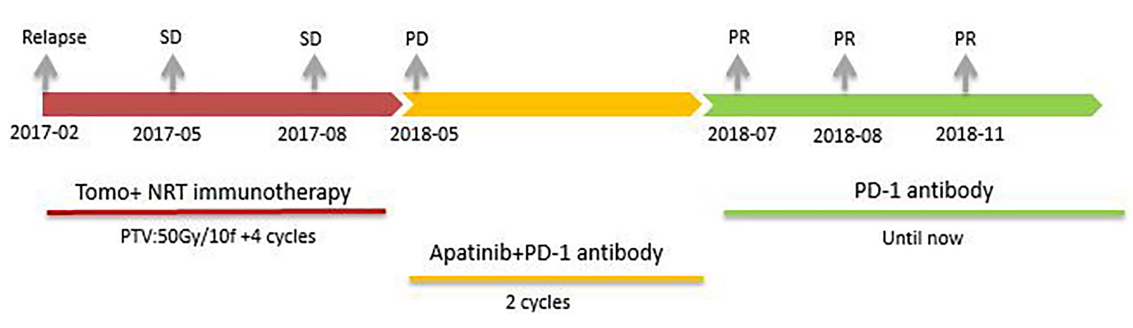

FIGURE 3 | The patient received different treatments at different times. Firstly, tomotherapy combined with Neoantigen Reactive T Cells based immunotherapy (2017-02 to 2017-08, SD). Secondly, PD-1 antibody combined with apatinib(2018-05 to 2018-07, PR). Finally, PD-1 antibody single-drug maintenance treatment (2018-07 until 2020-06, PR in porta hepatis and CR in left outer lobe).

there is no other special discomfort. But it brings significant survival benefits to patients.

\section{DATA AVAILABILITY STATEMENT}

The datasets presented in this study can be found in online repositories. The names of the repository/repositories and accession number(s) can be found in the article/supplementary material.

\section{ETHICS STATEMENT}

The studies involving human participants were reviewed and approved by Medical Ethics Committee of Drum Tower Hospital Affiliated to Nanjing University Medical School. The patients/ participants provided their written informed consent to participate in this study.

\section{REFERENCES}

1. Siegel RL, Miller KD, Jemal A. Cancer Statistics, 2018. CA Cancer J Clin (2018) 68:7-30. doi: 10.3322/caac.21442

2. Heimbach JK, Kulik LM, Finn RS, Sirlin CB, Abecassis MM, Roberts LR, et al. AASLD Guidelines for the Treatment of Hepatocellular Carcinoma. Hepatology (2018) 67(1):358-80. doi: 10.1002/hep.29086

3. European Association for the Study of the Liver. Easl Clinical Practice Guidelines: Management of Hepatocellular Carcinoma. J Hepatol (2018) 69(1):182236. doi: 10.1016/j.jhep.2018.03.019

4. Dhir M, Melin AA, Douaiher J, Lin C, Zhen WK, Hussain SM, et al. A Review and Update of Treatment Options and Controversies in the Management of Hepatocellular Carcinoma. Ann Surg (2016) 263:1112-25. doi: 10.1097/ SLA.0000000000001556

5. Qin S, Bai Y, Lim HY, Thongprasert S, Chao Y, Fan J, et al. Randomized, Multicenter, Open-Label Study of Oxaliplatin Plus Fluorouracil/Leucovorin

\section{AUTHOR CONTRIBUTIONS}

JShe and BL designed the clinical trial. CL, JShe and QX composed the manuscript and provided figures. CL, JSha, YD, QX, JY, JL, BL and JShe did the work of the acquisition, analysis and interpretation of the data. JShe, YD, ZZ, FC and SL revised the manuscript critically for important intellectual content, and agreement to be accountable for all aspects of the work, in ensuring that questions related to the accuracy or integrity of any part of the work are appropriately investigated and resolved. All authors ontributed to the article and approved the submitted version.

\section{FUNDING}

This study was supported by National Natural Science Foundation of China (No. 81902914); Jiangsu Provincial Medical Youth Talent (No. QNRC2016043).

Versus Doxorubicin as Palliative Chemotherapy in Patients With Advanced Hepatocellular Carcinoma From Asia. J Clin Oncol (2013) 31:3501-8. doi: 10.1200/JCO.2012.44.5643

6. Bhayani NH, Jiang Y, Hamed O, Kimchi ET, Staveley-O'Carroll KF, Gusani NJ. Advances in the Pharmacologic Treatment of Hepatocellular Carcinoma Curr Clin Pharmacol (2015) 10:299-304. doi: 10.2174/15748847106661 51020100059

7. Kanda T, Ogasawara S, Chiba T, Haga Y, Omata M, Y okosuka O. Current Management of Patients With Hepatocellular Carcinoma. World J Hepatol (2015) 7:1913-20. doi: 10.4254/wjh.v7.i15.1913

8. Phan GQ, Rosenberg SA. Adoptive Cell Transfer for Patients With Metastatic Melanoma: The Potential and Promise of Cancer Immunotherapy. Cancer Control (2013) 20:289-97. doi: 10.1177/107327481302000406

9. Tran E, Turcotte S, Gros A, R obbins PF, Lu YC, Dudley ME, et al. Cancer Immunotherapy Based on Mutation-Specific Cd4+T Cells in a Patient With Epithelial Cancer. Science (2014) 344:641-5. doi: 10.1126/science.1251102 
10. Schumacher TN, Schreiber RD. Neoantigens in Cancer Immunotherapy. Science (2015) 348:69-74. doi: 10.1126/science.aaa4971

11. Menon H, Remapriyan R, Cushman TR, Verma V, Kim HH, Schoenhals JE, et al. Role of Radiation Therapy in Modulation of the Tumor Stroma and Microenvironment. Front Immunol (2019) 10:193. doi: 10.3389/fimmu. 2019.00193

12. Ally A, Balasundaram M, Carlsen R, Chuah E, Clarke A, Dhalla N, et al. Cancer Genome Atlas Research Network. Comprehensive and Integrative Genomic Characterization of Hepatocellular Carcinoma. Cell (2017) 169:1327-41. doi: 10.1016/j.cell.2017.05.046

13. Xu JM, Shen J, Zhang Y, et al. 983p - Camrelizumab (C) in Combination With Apatinib (a) in Patients With Advanced Hepatocellular Carcinoma (RESCUE): An Open-Label, Multi-Center, Phase II Trial. Ann Oncol (2020) 31(suppl_4):S629-44. doi: 10.1016/j.annonc.2020.08.1099

14. Xu JM, Shen J, Gu SZ, Zhang Y, Wu LH, Wu J, et al. Radiotherapy: Changing the Game in Immunotherapy. Trends Cancer (2016) 2:286-94. doi: 10.1016/ j.trecan.2016.05.002

15. Herrera FG, Bourhis J, Coukos G. Radiotherapy Combination Opportunities Leveraging Immunity for the Next Oncology Practice. CA Cancer J Clin (2017) 67:65-85. doi: 10.3322/caac.21358

16. Weichselbaum RR, Liang H, Deng L, Fu YX. Radiotherapy and Immunotherapy: A Beneficial Liaison? Nat Rev Clin Oncol (2017) 14:36579. doi: $10.1038 /$ nrclinonc. 2016.211
17. Fukumura D, Kloepper J, Amoozgar Z, Duda DG, Jain RK. Enhancing Cancer Immunotherapy Using Antiangiogenics:Opportunities and Challenges. Nat Rev Clin Oncol (2018) 15:325-40. doi: 10.1038/nrclinonc.2018.29

18. Kato Y, Tabata K, Kimura T, Yachie-Kinoshita A, Ozawa Y, Yamada K, et al Lenvatinib Plus anti-PD-1 Antibody Combination Treatment Activates CD81 T Cells Through Reduction of Tumor-Associated Macrophage and Activation of the Interferon Pathway. PLoS One 14(2):e0212513, 201. doi: 10.1371/ journal.pone. 0212513

19. Finn RS, Ikeda M, Zhu AX, Sung MW, Baron AD, Kudo M, et al. Phase Ib Study of Lenvatinib Plus Pembrolizumab in Patients With Unresectable Hepatocellular Carcinoma. J Clin Oncol (2020) 38(26):2960-70. doi: 10.1200/JCO.20.00808

Conflict of Interest: The authors declare that the research was conducted in the absence of any commercial or financial relationships that could be construed as a potential conflict of interest.

Copyright $\odot 2021$ Liu, Shao, Dong, Xu, Zou, Chen, Yan, Liu, Li, Liu and Shen. This is an open-access article distributed under the terms of the Creative Commons Attribution License (CC BY). The use, distribution or reproduction in other forums is permitted, provided the original author(s) and the copyright owner(s) are credited and that the original publication in this journal is cited, in accordance with accepted academic practice. No use, distribution or reproduction is permitted which does not comply with these terms. 